\title{
Impact-parameter dependence of the electronic energy-loss of fast cluster projectiles
}

\author{
R.C. Fadanelli, P.L. Grande \\ Instituto de Física da Universidade Federal do Rio Grande do Sul, \\ Avenida Bento Gonçalves 9500, 91501 - 970, Porto Alegre, RS, Brazil \\ G. Schiwietz \\ Hahn-Meitner-Institut, Abteilung SF4 \\ Glienicker Str. 100, D-14109 Berlin, Germany
}

(Dated: July 10, 2007)

\begin{abstract}
Electronic energy loss of molecular clusters as a function of impact-parameter is less understood than atomic energy loss. Vicinage effects due to mutual interference between cluster fragments play a key role in the determination of the cluster electronic energy loss. In this work, we describe a molecular extension of the PCA (perturbative convolution approximation) energy-loss model, namely MPCA (molecular PCA), which yields remarkable agreement with first-order Born (SCA) results. The physical inputs of the model are the oscillators strengths of the target atoms and the projectile electron density. A very good agreement is obtained with time consuming full first-order calculations for bare incident molecular clusters for several angles between cluster axis and velocity direction.
\end{abstract}

PACS numbers: $34.50 . \mathrm{Bw}, 61.85 .+\mathrm{p}, 36.40 .-\mathrm{c}$ 


\section{INTRODUCTION}

Beams of molecules and ionic clusters are useful tools in both fundamental research and in material science and plasma physics. The effects of a cluster clearly deviate from the sum of individual effects of each cluster component. In particular, cluster-beam experiments were reported in mid 70s by Poizat and Remillieux [1] and, not much time after, the first evidence of the vicinage effect was reported by Brandt et. al.[2]. Since then, it is established that the cluster energy-loss is different from the sum of energy losses of its separated components. An increased energy transfer due a cluster may even be used in inertial nuclear fusion processes $[3,4]$.

If the ions enter along a principal axis of a crystalline target, their motion will be guided due the correlated collisions with the target atoms. These ions, then, are said to be channeled. The channeling motion of a molecule (or a cluster) will also depend on the Coulomb heating phenomenon (i. e., an increased transverse energy of the cluster fragments due to the mutual Coulomb repulsion), discovered in mid 70s by Caywood et. al. and Poizat et. al. $[5,6]$. Recently, the Coulomb heating was simulated $[7,8]$ and experimentally determined in a quantitative way [8].

The cluster stopping power can be theoretically treated by the united-atom model [9, 10], that describes the cluster as an equivalent single atom, with atomic number and mass being the sum of atomic number and mass of each component. However, that model is limited to the very beginning of the interaction between the cluster and the target, therefore, it cannot be used to understand several channeling key effects, for instance, the Coulomb heating effect. An important theoretical treatment is the dielectric formalism in a homogenous electron-gas target $[2,11]$ (a detailed review about vicinage effect and dielectric formalism for clusters can be found in ref.[12]) and, to account for the target-core effect, the LDA model (used for clusters in ref. [13]). However, although successful for homogeneous targets, the dielectric formalism cannot be used to easily describe the cluster energy-loss under channeling conditions, where the target cannot be treated as being homogeneous. Then, a theoretical investigation of the cluster stopping-power under channeling conditions, considering the Coulomb heating, requires the use of the impact parameter method, as presented by Jensen, Mikkelsen and Sigmund [14] but for distant collisions only.

This work describes an extension of the PCA model $[15,16]$, based on the impact parame- 
ter method, for molecules and clusters, namely MPCA (molecular perturbative convolution approximation). MPCA gives the energy loss as a function of impact parameter without time consuming first-order calculations using a set of thousands of final states for both distant and close collisions. The starting point of the model is the diatomic molecule. The physical inputs of the model are the target oscillator strengths, the target electronic density, the projectile screening function and the molecular alignment angles.

\section{MODEL}

The MPCA (Molecular Perturbative Convolution Approximation) model is an extension of PCA model $[15,16]$ made for cluster projectiles. Here, we present only a short outline of the PCA method but special attention will be draw to the interference terms that arise from the sum of all ionic potentials. The electronic energy loss is calculated from the expression

$$
Q(b)=\sum_{\beta}\left|a_{\beta}(\vec{b})\right|^{2}\left(\varepsilon_{\beta}-\varepsilon_{0}\right)
$$

which involves a sum of all final target states with energy $\varepsilon_{\beta}\left(\varepsilon_{0}\right.$ is the ground-state energy) and the corresponding calculation of all transition amplitudes $a_{\beta}$ for each cluster impact parameter $b$. In order to calculate the energy loss due to target ionization and excitation in a first order perturbation framework, we have to consider the amplitudes for each electronic transition between the initial state $|0\rangle$ to a final state $|\beta\rangle$ due to the the cluster with $N$ ions

$$
a_{\beta}(\vec{b})=-i \int_{-\infty}^{+\infty} d t e^{i\left(\varepsilon_{\beta}-\varepsilon_{0}\right) t}\left\langle\beta\left|\sum_{i=1}^{N} V_{i}\left(\vec{r}-\vec{R}_{i}(t)\right)\right| 0\right\rangle,
$$

where $V_{i}$ is the interacting potential between the $i$-th ion (whose charge is $Z_{i}$ ) in the cluster and the target electron. The $i$-th ion position in space is $\vec{R}(t)$ and $\vec{r}$ is the electronic coordinate, both relative to the target nucleous. In the first-order treatment the transition amplitude is only a coherent sum of amplitudes due to each ion of the cluster. If not indicated otherwise, all calculations throughout this work are in atomic units $\left(\hbar=m_{e}=e=1\right)$.

All calculations shall be done for a cluster projectile with impact parameter $\vec{b}$ with respect to the cluster center. For a straight-line projectile motion without vibrational and rotational degrees of freedom, the time dependent position of the $i$-th projectile nucleous is given by $\vec{R}_{i}(t)=\vec{b}_{i}+\vec{v} t+\vec{d}_{i z}$, where $\vec{d}_{i}$ is the distance between the molecule center and the $i$-th ion, 
$d_{i z}$ is the $z$-component of $\vec{d}_{i}, \vec{b}_{i}=\vec{b}+\vec{d}_{i \rho}$ is the $i$-th ion impact parameter and $\vec{v}$ is the cluster velocity. Figure 1 shows these vectors for a diatomic molecule.

The interacting potential $V_{i}$ may be one of the following (more details about these potentials are given in ref. [16]):

a) the Coulomb potential, that describes the potential induced by a moving point charge in vacuum;

b) the Bohr potential, that describes a potential produced by a external point charge immersed in a homogenous electron gas. The screening parameter $\left(\alpha_{i}\right)$ can be obtained either from the Debye screening length [17] or from the generalization of the Friedel sum rule for finite velocities derived by Lifschitz and Arista [18];

c) the single-zeta potential, that describes the potential due a projectile carrying one or two bound electrons $\left(n_{i}=1,2\right)$ in hydrogen-like 1s orbitals.

It is important to point out that not all ions of the cluster are necessarily generating the same kind of potential. Due to dynamic capture-loss processes, it is possible to find one ion of projectile cluster completely ionized, while its neighbors, after capturing an electron during ion-matter interactions, can have a single-zeta potential. This possibility must be taken into account in computer simulation codes.

According to the atomic PCA model, in a first step we shall find approximations for $Q(b)$ (Eq.(1)) that are valid for a limited range of impact parameters and in a second step these approximations should be linked. At large impact parameters the dipole approximation for $V_{i}$ can be used, and thus, an analytical expression [19, 20] for $Q(b)$ may be obtained. Inserting the cluster interacting potential, we have, for large impact parameters, an expression of the form

$$
Q^{\text {dipole }}(\vec{b})=\sum_{i=1}^{N} Q_{\text {atomic }}^{\text {dipole }}\left(\vec{b}_{i}\right)+\sum_{i=1}^{N} \sum_{j>i}^{N} Q_{\text {int }}^{\text {dipole }}\left(\vec{b}_{i}, \vec{b}_{j}\right)
$$

where

$$
Q_{\text {int }}^{\text {dipole }}\left(\overrightarrow{b_{i}}, \overrightarrow{b_{j}}\right)=\sum_{\beta} f_{\beta} \frac{2 Z_{i} Z_{j}}{v^{2}} \cos \left(\frac{\omega_{\beta 0} d_{i j z}}{v}\right)\left[\frac{2 \vec{b}_{i} \cdot \vec{b}_{j}}{\left(b_{i} b_{j}\right)^{2}} g_{\perp}\left(b_{i}\right) g_{\perp}\left(b_{j}\right)+\frac{2 g_{\|}\left(b_{i}\right) g_{\|}\left(b_{j}\right)}{b_{i} b_{j}}\right]
$$

where, for the Coulomb potential, the functions $g_{\|}\left(b_{i}\right)$ and $g_{\perp}\left(b_{i}\right)$ read:

$$
g_{\|}\left(b_{i}\right)=\left(\frac{\omega_{\beta 0} b_{i}}{v}\right) K_{0}\left(\frac{\omega_{\beta 0} b_{i}}{v}\right)
$$


and

$$
g_{\perp}\left(b_{i}\right)=\left(\frac{\omega_{\beta 0} b_{i}}{v}\right) K_{1}\left(\frac{\omega_{\beta 0} b_{i}}{v}\right)
$$

where $K_{0}(x)$ and $K_{1}(x)$ are second kind Bessel functions (for the Bohr and single-zeta potentials, expressions are given in ref. [16]), $f_{\beta}=2|\langle\beta|z| 0\rangle|^{2}\left(\varepsilon_{\beta}-\varepsilon_{0}\right)$ are the oscillator strenghts and $d_{i j z}=d_{i} \cos \theta_{i}-d_{j} \cos \theta_{j}$. The first term in Eq.(3) corresponds to the individual $Q_{\text {atomic }}^{\text {dipole }}(b)$ associated to each ion (see expressions in ref.[16]) and the last one is associated to interference effects (vicinage). The first interference term in Eq.(4), the one associated with $g_{\perp}$, corresponds to the classical sudden approximation. It is important to point out that the function $g_{\perp}\left(b_{i}\right)$ approaches 1 for small $b_{i}$ and $g_{\|}\left(b_{i}\right)$ approaches zero.

For small impact parameters, the influence of the target potential can be neglected at high projectile energies, allowing for an analytical expression for $Q^{\text {close }}(b)$ by replacing the final target-continuum states by plane waves. Thus, the energy transfer reads

$$
Q^{\text {close }}(\vec{b})=\sum_{i=1}^{N} Q_{\text {atomic }}^{\text {close }}\left(\vec{b}_{i}\right)+\int d^{2} r_{\perp} K_{\text {int }}^{\text {close }}\left(\vec{r}_{\perp}-\vec{b}\right) \int_{-\infty}^{\infty} d z \rho\left(\vec{r}_{\perp}, z\right)
$$

where again the first term corresponds to a incoherent sum of energy losses due to each ion from the cluster (already defined in ref [15] ) and

$$
K_{\text {int }}^{\text {close }}(\vec{b})=\frac{2}{v^{2}} \sum_{i=1}^{N} \sum_{j>i}^{N} Z_{j} Z_{i} h_{i n t}\left(\vec{b}_{j}, \vec{b}_{i}\right)
$$

is the interference term with

$$
\begin{aligned}
& h_{i n t}\left(\vec{b}_{j}, \vec{b}_{m}\right)=4 v^{2} \int_{0}^{1} d q q^{2} \times \\
& \cos \left(2 v q^{2} d_{m j z}\right)\left\{q\left[J_{0}\left(2 v q b_{j} \sqrt{1-q^{2}}\right) K_{0}\left(2 v q^{2} b_{m}\right)+J_{0}\left(2 v q b_{m} \sqrt{1-q^{2}}\right) K_{0}\left(2 v q^{2} b_{j}\right)\right]+\right. \\
& \left.\sqrt{1-q^{2}} \frac{\vec{b}_{j}}{b_{j}} \cdot \frac{\vec{b}_{m}}{b_{m}}\left[K_{1}\left(2 v q^{2} b_{m}\right) J_{1}\left(2 v q b_{j} \sqrt{1-q^{2}}\right)+K_{1}\left(2 v q^{2} b_{j}\right) J_{1}\left(2 v q b_{m} \sqrt{1-q^{2}}\right)\right]\right\}
\end{aligned}
$$

The function $h_{\text {int }}\left(\vec{b}_{1}, \vec{b}_{2}\right)$ approaches zero for $b_{1} \ll 1 / v$ or $b_{2} \ll 1 / v$ and, for large values of $b$ (i.e. large values for both $b_{1}$ and $b_{2}$ ), it reaches

$$
h_{\text {int }}\left(\vec{b}_{1}, \vec{b}_{2}\right) \approx 2 \frac{\vec{b}_{1} \cdot \vec{b}_{2}}{\left(b_{1} b_{2}\right)^{2}}
$$


recognized as the interference part of the classical sudden approximation result [20] for a diatomic molecule.

In what follows, we propose the following general formula, applicable to all impact parameters, namely

$$
Q(\vec{b})=\int d^{2} r_{\perp} K_{M P C A}\left(\vec{r}_{\perp}-\vec{b}\right) \int_{-\infty}^{\infty} d z \rho\left(\vec{r}_{\perp}, z\right)
$$

where the kernel is defined as

$$
K_{M P C A}(\vec{b})=K_{\text {atomic }}^{M P C A}(\vec{b})+K_{\text {int }}^{M P C A}(\vec{b})
$$

where $K_{\text {atomic }}^{M P C A}$ corresponds to the sum of the energy losses due to each individual ion from the cluster as presented in ref[15] for a single ion projectile and

$$
\begin{aligned}
& K_{\text {int }}^{M P C A}(\vec{b})=\sum_{\beta} f_{\beta} \sum_{i=1}^{N} \sum_{j>i}^{N} \frac{2 Z_{i} Z_{j}}{v^{2}} \cos \left(\frac{\omega_{\beta 0} d_{i j z}}{v}\right) \times \\
& {\left[h_{\text {int }}\left(2 v \vec{b}_{i}, 2 v \vec{b}_{j}\right) g_{\perp}\left(b_{i}\right) g_{\perp}\left(b_{j}\right)+\frac{2 g_{\|}\left(b_{i}\right) g_{\|}\left(b_{j}\right)}{\sqrt{b_{i}^{2}+b_{\min }^{2}} \sqrt{b_{j}^{2}+b_{\min }^{2}}}\right]}
\end{aligned}
$$

where $b_{\min }=1 / v^{2}$ is defined in ref [20]. This is the molecular perturbative convolution approximation (MPCA).

As can be observed the kernel function in Eq.(13) is based on the expression for large impact parameters $(\mathrm{Eq}(3))$ by replacing the interference term $\frac{2 \vec{b}_{i} \cdot \vec{b}_{j}}{\left(b_{i} b_{j}\right)^{2}}$ by $h_{i n t}\left(b_{i}, b_{j}\right)$. In this way, according to Eq.(10), the above energy-loss ansatz interpolates smoothly small and large impact parameters.

In figure 2, we compare the dipole and close-collision interference terms with the corresponding MPCA term for two bare diatomic molecule orientations. For both orientations, we can see that MPCA and close-collision interference terms (from Eq.(13) and Eq.(8), respectively) agree with each other for small impact parameters and the same is observed between MPCA and dipole approximations (from Eq.(13) and Eq.(4) for large impact parameters, thus reinforcing the validity of our proposed general formula (11). It should be stressed that the sudden approximation, which was used in ref. [15] to link close and distant collisions for atomic projectiles, in fact does not link the interference terms for close and distant collisions properly. This comes from the fact that the classical sudden approximation does not contain the interference terms due to the phase difference along the z-direction (e.g. the cosine term in Eq.(4) and in Eq.(9)). 
It is important to point out that Eq. (11) is valid only for a one-electron system. In the framework of the independent particle model, however, it is possible to use eq.(11) considering the electronic density and the dipole oscillator strengths for each electron of all occupied target shells.

In what follows, only an analysis of the interference term will be performed. The corresponding analysis of the monoatomic terms was already done in ref. [15]. The angles $\theta$ and $\phi$ shown in figure 1 will fix the diatomic molecular orientation.

\section{DISCUSSIONS AND CONCLUSIONS}

In fig. 3 we see the results of the present model, for two molecule orientations (where $\phi=0^{\circ}$ ), for the impact parameter dependence of the mean energy loss of bare (top) and single-zeta screened (bottom) $\mathrm{H}_{2}$ molecular projectiles, both at $500 \mathrm{keV} / \mathrm{amu}$, colliding with atomic H (full line). We compare our results with full first order molecular SCA (semiclassical approximation, similar to the numerical procedure seen in [21] calculations (squares) and with full first order SCA for two independent protons with the same screen function and impact parameters as used in molecular SCA (dashed line). In our tests, the interatomic distance was set to 2 a. u. (about $1.06 \AA$ ).

About 3500 target states were used in SCA calculations, to ensure an adequate number of partial waves, necessary to calculate $Q(b)$ accurately. Here we have considered two cases. The first one the molecule has no bound electrons (two protons traveling together, interacting with Coulomb forces) and the second one where one of the proton has captured one electron from the medium $\left(\mathrm{H}^{+}\right.$and $\mathrm{H}^{0}$ traveling together).

Fig. 3 (on the top panel) shows a fairly good agreement between molecular SCA and MPCA model. Moreover, it is possible to appreciate the interference terms effect, shown by the difference between MPCA and independent-protons SCA. For $\theta=90^{\circ}$ and $\phi=0^{\circ}$ (i.e. , the molecule has its axis orthogonal to its motion and parallel to the impact parameter direction), the increase of energy loss up to impact parameter about $1 \mathrm{a}$. $\mathrm{u}$. is due the choice of the coordinated system. In that orientation and for $b=1$ a. u., one of the ions (namely the second) will have a head-on collision with the target. In all orientations, the effect of interference terms leads to an increase in energy loss of about $50 \%$ for distant collisions and less than $10 \%$ for close collisions. That result agrees with the united atom model for 
distant collisions, where the energy loss is proportional to $\left(Z_{1}+Z_{2}\right)^{2}\left(4\right.$, for $\left.\mathrm{H}_{2}^{+}\right)$and the independent atom model for close collisions, where $S_{c}$ is proportional to $Z_{1}^{2}+Z_{2}^{2}\left(2\right.$, for $\left.\mathrm{H}_{2}^{+}\right)$.

Fig. 3 (on the bottom panel) shows similar results for a molecule projectile, whose first ion has a single-zeta screening with $\alpha=2\left(Z_{e f f}=1\right)$. It is pointed out that the interference between the projectile components is notably reduced, since the characteristic screening length $1 / \alpha$ is only one quarter of the molecule length. Then, the interaction between the bare ion and the target electron is much larger than the one from the screened partner. This explains the significantly reduced difference between molecular SCA and independent proton SCA calculations for screened projectiles. Qualitatively similar results were found for different values of $\phi$ and for the case of Bohr screening for $\alpha=2$ (not shown).

In conclusion, we have developed a simple formula (Eqs.(11-13)) to evaluate the electronic energy loss as a function of impact parameter for cluster projectiles, valid for high clusters energies and for a wide range of impact parameters, including the effect of screening. The input parameters are only the target density and the oscillator strengths, as well as the projectile screening parameter for all cluster components. This model reproduces the results of full SCA calculations and is much less time consuming. Thus, the MPCA model is very adequate for use in computer channeling simulations.

\section{ACKNOWLEDGEMENTS}

This work was partially supported by Conselho Nacional Científico e Tecnológico (CNPq) and by Coordenação de Aperfeiçoamento de Pessoal de Nível Superior (CAPES).

[1] J.C. Poizat and J. Remillieux, Phys. Lett. 34A (1971) 53.

[2] W. Brandt, A. Ratkowski and R. H. Ritchie, Phys. Rev. Lett. 33 (1974) 1325.

[3] C. Deutsch, Laser Part. Beams 8 (1990) 541; C. Deutsch, Laser Part. Beams 10 (1992) 355; C. Deutsch and N.A. Tahir, Phys. Fluids B 4 (1992) 3735.

[4] R. J. Beuhler, G. Friedlander and L. Friedman, Phys. Rev. Lett. 63 (1989) 1292.

[5] J.M. Caywood, T.A. Tombrello and T.A. Weaver, Phys. Lett. 37A (1971) 350; T.A. Tombrello and J.M. Caywood Phys. Rev. B 8 (1973) 3065. 
[6] J.C. Poizat and J. Remillieux J. Phys. B: Atom. Molec. Phys. 5 (1972) L94.

[7] V.A. Khodyrev, V.S. Kulikauskas and C. Yang, Nucl. Instr. Meth. Phys. Res. B 195 (2002) 259.

[8] R.C. Fadanelli, P.L. Grande, M. Behar, J.F. Dias, G. Schiwietz and C. D. Denton, Phys. Rev. B (2004) (in press).

[9] D. Ben-Hamu et. al., Phys. Rev. A, 56 (1997) 4786.

[10] P. Sigmund, I. S. Bitensky and J. Jensen, Nucl. Instr. and Meth. B, 112 (1996) 1.

[11] N. R. Arista. Phys. Rev. B, 18, (1978) 1.

[12] N. R. Arista, Nucl. Instr. Meth. Phys. Res. B 164-165 (2000) 108.

[13] Y. N. Wang and T. C. Ma, Phys. Rev. A 50 (1994) 3192.

[14] J. Jensen, H. H. Mikkelsen and P. Sigmund, Nucl. Instr. and Meth. B, 88 (1994) 191.

[15] P. L. Grande and G. Schiwietz, Phys. Rev. A, 58, 3796 (1998)

[16] G. de M. Azevedo, P. L Grande and G. Schiwietz, Nuc. Instr. and Meth. B, 164, 203 (2000)

[17] M. G. Calkin and P. J. Nicholson, Rev. Mod. Phys. 39, 361 (1967)

[18] A. F. Lifschitz and N. R. Arista, Phys. Rev. A, 57, 200 (1998)

[19] C.O. Reinhold, J. Burgdoerfer, J. Phys. B: At. Mol. Opt. Phys. 26 (1993) 3101.

[20] J.D Jackson, Classical Electrodynamics, Wiley, 1975, Chapter 13.

[21] G. Schiwietz, Phys. Rev. A 42, 296 (1990); G. Schiwietz and P. L. Grande, Nucl. Instrum. Methods Phys. Res. B 69, 10 (1992); P. L. Grande and G. Schiwietz, Phys. Rev. A 47, 1119 (1993); P. L. Grande and G. Schiwietz, Nucl. Instrum. Methods Phys. Res. B 132, 264 (1997).

\section{Figure captions}

Figure 1. Representation of the collision geometry, showing the target nucleus, the target electronic distribution, the projectile nuclei, the impact parameter vectors and the projectile velocity.

Figure 2. Comparison between the MPCA model (solid lines), close-collision (dotted lines) and dipole-approximation (dashed lines) interference terms for bare molecules for two possible molecule orientations. In both orientations, we can see an accordance between MPCA and close-collision approximations for small impact parameters as well as between MPCA and dipole approximations for large impact parameters. 
Figure 3. Comparison between MPCA model and full first order SCA calculations for $500 \mathrm{keV} / \mathrm{amu}$ bare (on top panel) and single-zeta screened (on bottom panel) $\mathrm{H}_{2}$ projectile colliding with $\mathrm{H}$ target. The full lines stand for energy loss as a function of impact parameter as given by the MPCA (eq. 11) model. The squares stand for molecular SCA calculations and, to show the interference effect, the dashed lines stand for independent protons SCA calculation. 


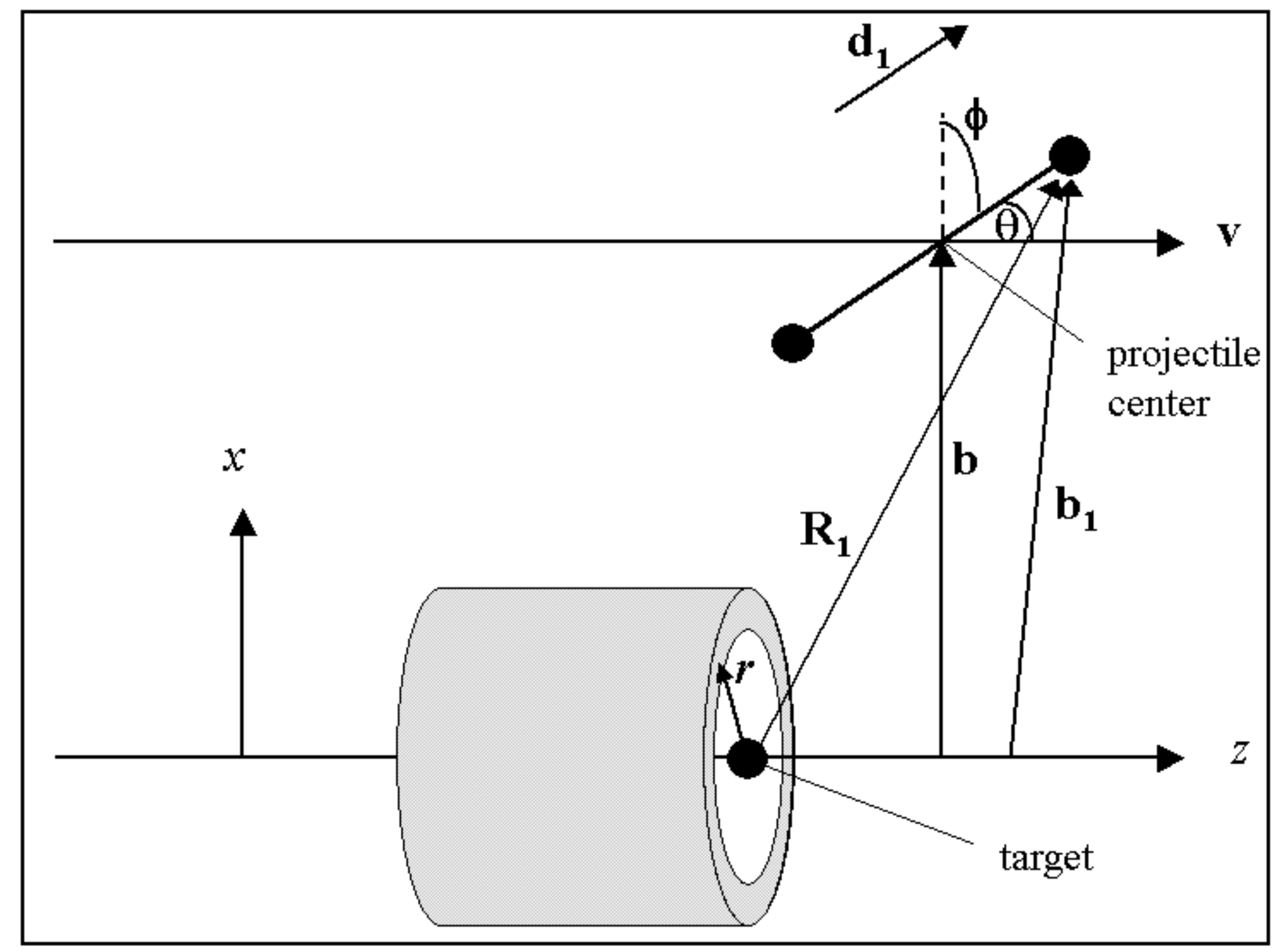




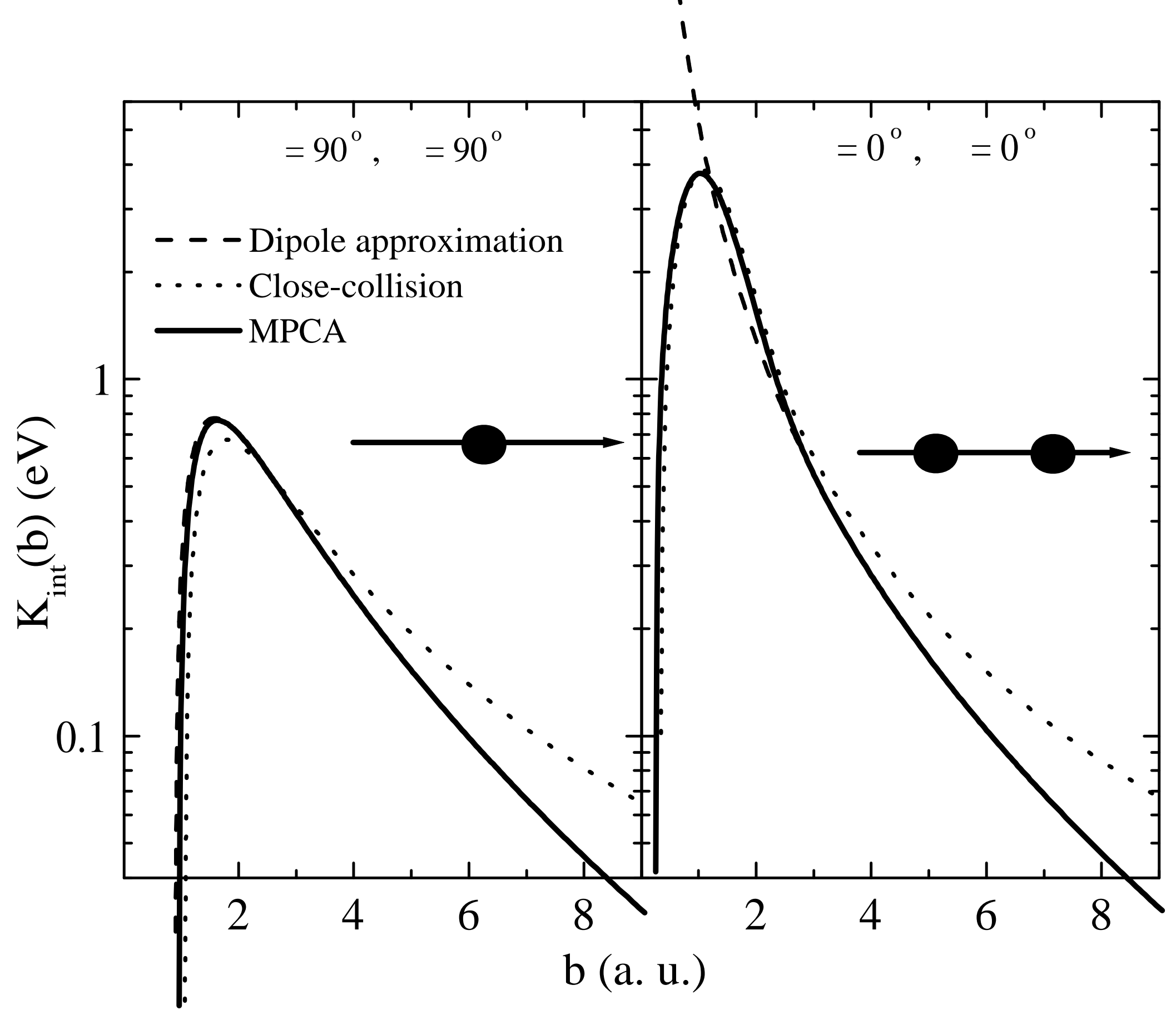




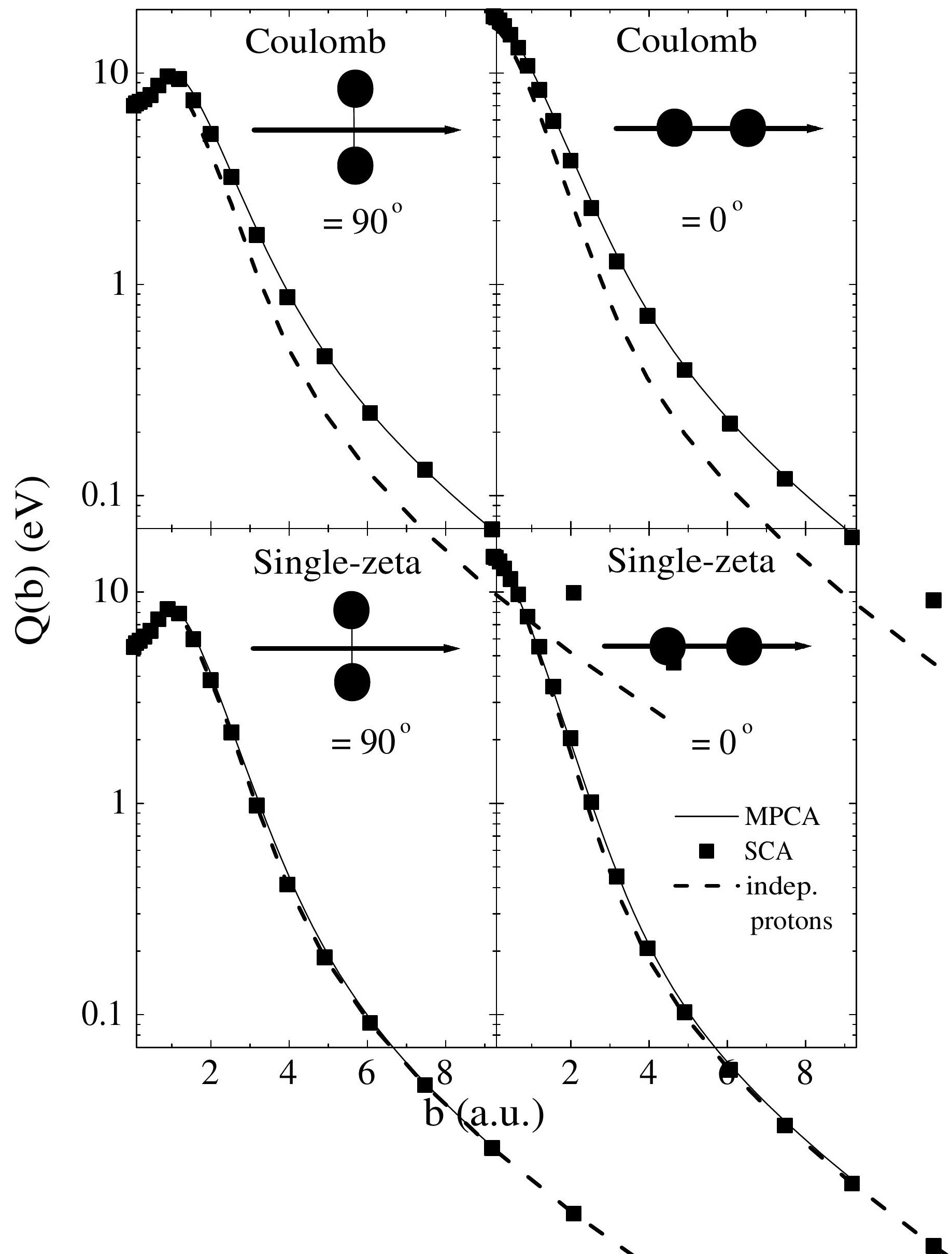

